\title{
Quantum Statistics and Slow Neutron Scattering by Gases*
}

\author{
J. P. PlumMer $\dagger$ † \\ Department of Physics, Queens College, City University of New York, Flushing, New York \\ AND \\ G. C. SuMMerfield $\dagger$ And P. F. Zweifel \\ Department of Nuclear Engineering, The University of Michigan, Ann Arbor, Michigan
}

(Received 5 January 1967)

\begin{abstract}
A surprisingly simple expression in "closed form" for the cross section $d^{2} \sigma / d \Omega d \epsilon$ for the scattering of thermal neutrons (including polarized neutrons) from an ideal quantum gas is derived. This result extends the work of Van Hove on the quantum gas. An expansion is obtained for $d \sigma / d \epsilon$. The case of elastic scattering is treated separately. From these expressions is obtained a criterion for ignoring the statistics of the scatterer in favor of classical (Boltzmann) statistics. This criterion should have some validity for weakly interacting systems. It is shown that the effects of statistics on the neutron cross section for a helium-4 gas range from $5 \%$ or less for the noninteracting gas up to as much as $40 \%$ for the interacting system.
\end{abstract}

\section{INTRODUCTION}

In most theoretical analyses of slow-neutron-scattering experiments, the wavefunctions of the scattering system are neither symmetrized nor antisymmetrized. The well-known exceptions to this rule are molecular hydrogen and liquid helium for which the statistics of the scatterer play a dominant role. ${ }^{1}$ Also, recent calculations ${ }^{2}$ indicate that for neutron scattering from methane at $10^{\circ} \mathrm{K}$, statistical corrections may be as great as $30 \%$. For most scatterers, however, very elementary arguments show that the application of quantum statistics yields only a small correction. However, such effects as the Kohn anomaly, crystal defects, and polarization effects in magnetic scattering-which are being measured-may produce structure in the neutron cross section amounting to only a fraction of a percent of the total cross section. It then becomes important and interesting to determine exactly how small the effects of the quantum statistics of the scatterer are in determining the neutron cross section.

The approximation made in ignoring statistics can be justified for many scatterers by noting that the number of phase space cells available to an atom is much greater than the number of atoms. This condition applied to an ideal gas can be expressed by the inequality $\eta \ll 1$, where the "figure of merit" $\eta$ is defined by $^{3}$

$$
\eta=\left[\rho /\left(2 S_{0}+1\right)\right]\left(h^{2} / 2 \pi M k T\right)^{3 / 2} .
$$

Here, $\rho$ is the number of identical atoms per unit

* This work was supported in part by the U.S. Atomic Energy Commission.

t Part of this work was done as temporary employees of Idaho Nuclear Corporation, Idaho Falls, Ida. 83401.

t Present address: State University of New York, New Paltz, N.Y.

${ }_{1}$ M. Nelkin, Phys. Rev. 127, 979 (1962); W. E. Parry and R. E. Turner, Ann. Phys. (N.Y.) 17, 301 (1962); J. A. Young and J. U. Koppel, Phys. Rev. 135, A603 (1964).

'S. K. Sinha and G. Venkataraman, Phys. Rev. 149, 1 (1966); P. Michael, ibid. 138, A692 (1965).

P. M. Morse, Thermal Physics (W. A. Benjamin, Inc., New York, 1964). volume in the scatterer, $M$ is the mass of an atom, $T$ is the absolute temperature, and $S_{0}$ is the nuclear spin. For $\eta \gg 1$, the identical particles obey quantum statistics. Thus, values of $\eta$ of order unity characterize the transition region in which the statistics of the system go from classical (Maxwell-Boltzmann) to quantum.

While $\eta$ is a quantitative measure of "how classical" a system of noninteracting identical particles is, it does not offer a direct measure of the effect of the inclusion of the correct statistics on the neutron cross section. In this paper we examine the question of the need for proper symmetrization of the wavefunctions by carefully treating the ideal-gas scattering system, thus isolating the effect of statistics from the complications of interactions. An expression in "closed form" for the cross section for the scattering of thermal neutrons from an ideal quantum gas (an ideal gas of bosons or fermions) is obtained. It is shown how to modify the expression to describe the scattering of polarized neutrons. These results extend the work of Placzek ${ }^{4}$ and Van Hove. ${ }^{5}$ The most useful results obtained by Van Hove for the quantum gas are in the form of expansions for the time-dependent pair distribution function. These expansions suffer from convergence problems as the region in which quantum statistics dominate is approached. Furthermore, Van Hove assumes an unpolarized incident beam.

From our result, we obtain a criterion for ignoring the effect of statistics on the cross section. This criterion should apply, at least semiquantitatively, to weakly interacting systems as well. As an example of the importance of statistics, we consider a system of gaseous ${ }^{4} \mathrm{He}$ atoms.

\section{THE NEUTRON CROSS SECTION}

Assuming only the Fermi approximation, and nothing about the statistics of the scattering system, the neutron

G. Placzek, Proc. Symp. Math. Statistics Probability 2nd Berkeley, Calif., 69 (1951).

${ }^{6}$ L. Van Hove, Phys. Rev. 95, 249 (1954). 
cross section is

$$
\frac{d^{2} \sigma}{d \Omega d \epsilon}=(2 \pi \hbar)^{-1} \frac{k_{f}}{k_{i}} \int_{-\infty}^{\infty} d t \exp \left(-i \frac{\epsilon}{\hbar} t\right)\left\langle\sum_{j l} a_{j}^{\dagger} a_{l} \exp \left[-i \mathrm{Q} \cdot \mathbf{r}_{j}(0)\right] \exp \left[i \mathrm{Q} \cdot \mathbf{r}_{l}(t)\right]\right\rangle_{T},
$$

where $\hbar \mathbf{k}_{f}$ and $\hbar \mathbf{k}_{i}$ are the final and initial momenta of the neutron, respectively,

$$
\mathrm{Q}=\mathbf{k}_{i}-\mathbf{k}_{f}
$$

and

$$
\epsilon=\left(\hbar^{2} / 2 m\right)\left(k_{i}{ }^{2}-k_{f}{ }^{2}\right)
$$

are the momentum and energy losses of the neutron, respectively; $m$ is the neutron mass; $r_{j}(0)$ and $r_{l}(t)$ are Heisenberg position operators for the $j$ th and $l$ th scattering nuclei, respectively; and $a_{j}$ and $a_{l}$ are boundatom scattering lengths (the matrix elements of which have already been taken between neutron spin states), assumed to be of the following spin-dependent form:

$$
a_{j}=b_{1}\left\langle\chi_{f} \mid \chi_{i}\right\rangle+b_{2} \mathbf{0} \cdot \mathbf{S}_{j},
$$

where $\chi_{f}$ and $\chi_{i}$ are the final and initial neutron spin states, respectively; $\mathbf{S}_{j}$ is the spin operator for the $j$ th nucleus; $\mathbf{d}=\left\langle\chi_{j}|\mathbf{s}| \chi_{i}\right\rangle$, where $\mathbf{s}$ is the neutron spin operator; and the coefficients $b_{1}$ and $b_{2}$ are the same constants for all the nuclei. The thermal average over initial states $|i\rangle$ of the scattering system is defined by

$$
\langle\rangle_{T} \equiv \sum_{i}\left[\exp \left(-\beta E_{i}\right) / \sum_{j} \exp \left(-\beta E_{j}\right)\right]\langle i|| i\rangle,
$$

where $E_{i}$ is the energy of the state $|i\rangle$ and $\beta=(k T)^{-1}$.

If the thermal average is evaluated in the "second quantization" representation, 7 the task of restricting the states $|i\rangle$ to totally symmetric or totally antisymmetric states is greatly simplified. The details of this calculation, leading to Eqs. (4) and (7), are presented in the Appendix in somewhat sketchy fashion, since nothing new is involved. (For a more complete presentation, see Ref. 8.) The result, for noninteracting bosons (upper signs) not in the condensed phase and for all noninteracting fermions (lower signs), ${ }^{9}$ is

$$
d^{2} \sigma_{ \pm} / d \Omega d \epsilon
$$

$$
\begin{array}{r}
=\left(k_{f} / k_{i}\right)\left[\left|b_{1}\right|^{2}\left|\left\langle\chi_{f} \mid \chi_{i}\right\rangle\right|^{2}+\left|b_{2}\right|^{2}|\boldsymbol{\delta}|{ }^{21} S_{0}\left(S_{0}+1\right)\right] \\
\times \sum_{\mathbf{K}} \delta\left(E_{\mathrm{K}+Q}-E_{\mathrm{K}}-\epsilon\right) f_{\mathrm{K}}{ }^{\mp}\left[1 \pm\left(2 S_{0}+1\right)^{-1} f_{\left.\mathrm{K}+Q^{\mp}\right]}\right] \\
+\left.N^{2}\left|b_{\mathbf{1}}{ }^{2}\right|\left\langle\chi_{f} \mid \chi_{i}\right\rangle\right|^{2} \delta(\mathrm{Q}) \delta(\epsilon),
\end{array}
$$

where

$$
E_{\mathrm{K}}=\hbar^{2} K^{2} / 2 M
$$

where $\hbar \mathbf{K}$ is an allowed value of nuclear momentum and $f_{\mathrm{K}}{ }^{\mp}$ is the usual Bose-Einstein (BE) or FermiDirac (FD) discrete space distribution function

$$
f_{\mathrm{K}}^{\mp}=\left(2 S_{0}+1\right) /\left[B \exp \left(\beta E_{\mathrm{K}}\right) \mp 1\right] .
$$

We have assumed there is no external magnetic field in the scattering system. The constant $B$ is determined by the condition

$$
\sum_{\mathbf{K}} f_{\mathbf{K}}^{\mp}=N
$$

where $N$ is the total number of scattering atoms. $B$ is completely fixed by the choice of statistics (BE or FD) and the selection of $\eta$. This can be seen from the fact that Eq. (6) can be put in the form

$$
\eta=\frac{2}{\sqrt{\pi}} \int_{0}^{\infty} \frac{u^{1 / 2} d u}{B e^{u} \mp 1}
$$

which yields $B(\eta)$, of course, only by application of numerical methods.

The result for the $\mathrm{BE}$ gas in the condensed phase (a BE gas is not in the condensed phase if $N_{0} / N \ll 1$, where $N_{0}$ is the number of bosons in the zero energy states; in terms of $\eta$, condensation exists for $\eta \geq 2.612)^{3}$ is given by a different expression. This is because certain relations for averages of products of occupation numbers for two cells in phase space are altered when $N_{0}$ becomes a nonnegligible fraction of $N$. The result for the condensed-phase $\mathrm{BE}$ ideal gas is

$$
\begin{gathered}
d^{2} \sigma / d \Omega d \epsilon=\left(k_{f} / k_{i}\right)\left[\left|b_{1}\right|^{2}\left|\left\langle\chi_{f} \mid \chi_{i}\right\rangle\right|^{2}+\left|b_{2}\right|^{2}|\sigma|^{2} \frac{1}{3} S_{0}\left(S_{0}+1\right)\right] \sum_{\mathrm{K}} \delta\left(E_{\mathrm{K}+\mathrm{Q}}-E_{\mathrm{K}}-\epsilon\right) f_{\mathrm{K}}\left[1+\left(2 S_{0}+1\right)^{-1} f_{\mathrm{K}+\mathrm{Q}}\right] \\
+\delta(\mathrm{Q}) \delta(\epsilon)\left\{\left[N_{0}^{2} /\left(2 S_{0}+2\right)\right]\left[\left|b_{1}\right|^{2}\left|\left\langle\chi_{f} \mid \chi_{i}\right\rangle\right|^{2}+\left|b_{2}\right|^{2}|\sigma|^{2 \frac{1}{3}} S_{0}\left(S_{0}+1\right)\right]\right. \\
\left.+\left[N^{2}-N_{0}^{2}\left(2 S_{0}+2\right)^{-1}\right]\left|b_{1}\right|^{2}\left|\left\langle\chi_{f} \mid \chi_{i}\right\rangle\right|^{2}\right\}
\end{gathered}
$$

where

$$
f_{\mathrm{K}}=\left(2 S_{0}+1\right) /\left[\exp \left(\beta E_{\mathrm{K}}\right)-1\right]
$$

\footnotetext{
'See, for example, R. J. Glauber, in Lectures in Theoretical Physics (Interscience Publishers, Inc., New York, 1962), Vol. 4. "See D. Falkoff, "The N-Body Problem," in The Many Body Problem, Christian Fronsdal, Ed. (W. A. Benjamin, Inc., New York, 1962).

8 (a) See J. P. Plummer, thesis, The University of Michigan, 1964; (b) J. P. Plummer, Idaho Nuclear Corp. Rept. (unpublished).

- This sign convention is observed throughout this paper.
} 
and

$$
\sum_{\mathbf{K} \neq 0} f_{\mathrm{K}}=N-N_{0} .
$$

To obtain the results for the case in which the incident neutron beam is unpolarized and the final neutron polarization is not measured, it is necessary to sum Expressions (4) and (7) over neutron final spin states and average (with the probabilities for spin "up" and for spin "down" each $\frac{1}{2}$ ) them over neutron initial spin states. One can quickly see that this results in

and

$$
\left|\left\langle\chi_{j} \mid \chi_{i}\right\rangle\right|^{2}=1
$$

$$
|d|^{2}=\frac{3}{4}
$$

It is then possible to express all factors in Eqs. (4) and (7) stemming from the spin-dependent scattering length in terms of the averages over spin states of the scattering length and of the square of the scattering length. For unpolarized neutrons and zero magnetic field, these $\operatorname{are}^{10}$

and

$$
\langle a\rangle=\left|b_{1}\right|
$$

$$
\left\langle a^{2}\right\rangle=\left|b_{1}\right|^{2}+\frac{1}{4}\left[S_{0}\left(S_{0}+1\right)\right]\left|b_{2}\right|^{2} .
$$

The resulting cross section for the scattering of unpolarized slow neutrons from an ideal gas of fermions (lower signs) or bosons not in the condensed phase (upper signs) is

$$
\begin{aligned}
d^{2} \sigma_{ \pm} / d \Omega d \epsilon= & \left(k_{f} / k_{i}\right)\left\langle a^{2}\right\rangle \sum_{\mathrm{K}} \delta\left(E_{\mathrm{K}+\mathrm{Q}}-E_{\mathrm{K}}-\epsilon\right) \\
& \times f_{\mathrm{K}}{ }^{\mp}\left[1 \pm\left(2 S_{0}+1\right)^{-1} f_{\mathrm{K}+\mathrm{Q}}{ }^{\mp}\right]+N^{2}\langle a\rangle^{2} \delta(\mathrm{Q}) \delta(\epsilon)
\end{aligned}
$$

and for bosons in the condensed phase,

$$
\begin{aligned}
& d^{2} \sigma / d \Omega d \epsilon=\left(k_{f} / k_{i}\right)\left\langle a^{2}\right\rangle \\
& \quad \times \sum_{\mathrm{K}} \delta\left(E_{\mathrm{K}+\mathrm{Q}}-E_{\mathrm{K}}-\epsilon\right) f_{\mathrm{K}}\left[1+\left(2 S_{0}+1\right)^{-1} f_{\mathrm{K}+\mathrm{Q}}\right] \\
& \quad+\delta(\mathbf{Q}) \delta(\epsilon)\left\{\left[N_{0}^{2} /\left(2 S_{0}+2\right)\right]\left(\left\langle a^{2}\right\rangle-\langle a\rangle^{2}\right)+N^{2}\langle a\rangle^{2}\right\} .
\end{aligned}
$$

Previous work ${ }^{5}$ on ideal quantum gases consisted of calculating the Van Hove spin-dependent pair correlation function for the quantum gas. This correlation function is

$$
\begin{array}{r}
\Gamma(\mathbf{r}, t)=(2 \pi)^{-3} N^{-\mathbf{1}} \int \exp [i(\omega t-\mathbf{Q} \cdot \mathbf{r})] \\
\times S(\mathbf{Q}, \omega) d^{3} \mathbf{Q} d \omega,
\end{array}
$$

where $\hbar \omega=\epsilon$ and the "scattering law" $S(\mathrm{Q}, \omega)$ is related to the cross section by

$$
d^{2} \sigma / d \Omega d \epsilon=\left(m^{2} k_{f} / 4 \pi^{2} \hbar^{5} k_{i}\right) S(\mathbf{Q}, \omega) .
$$

${ }^{10}$ See, for example, S. Yip, R. K. Osborn, and C. Kikuchi, "Neutron Acoustodynamics," University of Michigan Rept. No. IP-524, Chap. 8 (1961).
Substitution of the scattering laws implied by Eqs. (8) and (9) into Expression (10) yield exactly ${ }^{11}$ Van Hove's results for $\Gamma(\mathbf{r}, t)$. The terms in Eqs. (8) and (9) involving $\delta(\mathbf{Q}) \delta(\epsilon)$ - the first a Kronecker delta function, the second a Dirac delta function-represent forward scattering and are therefore unobservable. They are included in the expressions for the cross section because they give rise to the constant terms in the correlation functions. The function $\Gamma(\mathbf{r}, t)$ is a function of $r$ for a quantum gas, of course, in contrast to the ideal Boltzmann gas which has no interparticle correlations. The symmetrization of the ideal-gas wavefunctions introduces correlations among the scattering nuclei and hence interference effects in the neutron cross section.

The correlation function $\Gamma(\mathbf{r}, t)$ cannot be completely expressed in terms of functions which are tabulated. Van Hove obtains a series expansion in powers of $B^{-1}$ for $\Gamma(\mathbf{r}, t)$. Since the quantum limit is $B=1$ for bosons and $B=0$ for fermions, such an expansion is not very suitable for investigating the effects of statistics on neutron scattering. However, we point out at this time that the cross section can be expressed in "closed form." Ignoring forward scattering, Expression (8) for the cross section per atom ${ }^{12}$ can be shown to be (except for bosons in the condensed phase)

$$
\begin{array}{r}
\frac{d^{2} \sigma_{ \pm}}{d \Omega d \epsilon}=\mp \frac{1}{2}\left\langle a^{2}\right\rangle\left[\frac{\beta}{\pi E_{\mathrm{Q}}}\right]^{1 / 2} \frac{k_{f}}{k_{i}} \eta^{-1}[\exp (-\beta \epsilon)-1]^{-1} \\
\times \ln \left\{\frac{1 \mp B^{-1} \exp [-(\alpha+\beta \epsilon)]}{1 \mp B^{-1} e^{-\alpha}}\right\},
\end{array}
$$

where

$$
\alpha=\left(\beta / 4 E_{Q}\right)\left(E_{Q}-\epsilon\right)^{2} .
$$

Despite the seeming menace of the minus sign in front of the entire expression for $\mathrm{BE}$ statistics, the cross section can be shown to be a positive definite expression. The cross section (per atom) for thermal neutron scattering from an ideal Boltzmann gas has been derived many times ${ }^{8 \mathrm{a}}$ and is

$$
d^{2} \sigma_{c} / d \Omega d \epsilon=\frac{1}{2}\left\langle a^{2}\right\rangle\left[\beta / \pi E_{Q}\right]^{1 / 2}\left(k_{f} / k_{i}\right) e^{-\alpha} .
$$

It is easy to show that $\left(d^{2} \sigma_{ \pm} / d \Omega d \epsilon\right) \rightarrow\left(d^{2} \sigma_{c} / d \Omega d \epsilon\right)$ in the limit of classical statistics. The most direct way of verifying this is to note first that in the classical limit,

$$
\eta B \rightarrow 1
$$

(the proof of this is not difficult). Thus, one merely sets $B=\eta^{-1}$ in Eq. (12) and lets $\eta \rightarrow 0$ (or, alternatively, sets $\eta=B^{-1}$ and lets $\left.B \rightarrow \infty\right)$ and Eq. (13) evolves.

\footnotetext{
11 There is an apparent oversight in Ref. 5 . In the definition of a quantity called $n_{ \pm}(r, t)$, and later $n(r, t)$ for the condensedphase $B E$ gas, a factor of $\left(2 S_{0}+1\right)$ is missing. Also Van Hove absorbs a factor of $(2 \pi \hbar / \mathrm{m})$ into his definition of the scattering length $a$.

${ }_{12}$ Equations ( 8 ) and (9) must be divided by $N$, the number of scatterers, to give the cross section per atom.
} 
While Eq. (12) cannot be integrated over all $\boldsymbol{\Omega}$ such that the result is expressible in terms of tabulated functions, it is possible to expand the integrand (the resulting series converges uniformly for $B>1$ and thus may be integrated term by term) in powers of $B^{-1}$ and then relate the coefficient integrals to the classical cross section. The result is

$$
\begin{aligned}
\frac{d \sigma_{ \pm}}{d \epsilon}=(\eta B)^{-1}\left[\frac{d \sigma_{c}}{d \epsilon} \pm\left(2^{3 / 2} B\right)^{-1}\right. & {[\exp (-\beta \epsilon)+1]\left(\frac{d \sigma_{c}}{d \epsilon}\right)_{\beta \rightarrow 2 \beta} } \\
+\left(3^{3 / 2} B^{2}\right)^{-1}[\exp (-2 \beta \epsilon) & +\exp (-\beta \epsilon)+1] \\
& \left.\times\left(\frac{d \sigma_{c}}{d \epsilon}\right)_{\beta \rightarrow 3 \beta} \pm \cdots\right],
\end{aligned}
$$

where

$$
\frac{d \sigma_{c}}{d \epsilon}=(\pi \beta)^{1 / 2}\left\langle a^{2}\right\rangle \frac{k_{f}}{k_{i}} \int_{-1}^{1} E_{Q^{-1 / 2}} e^{-\alpha} d \mu .
$$

The notation $\beta \rightarrow \eta \beta$ implies the replacement of $\beta$ by $\eta \beta$ in equation (15) in which $\mu=\cos \theta$ and $\theta$ is the scattering angle. The $\mu$ dependence of the integrand in Eq. (15) comes through $E_{Q}$. Both expressions (14) and (15) give the cross section per atom. Wigner and Wilkins ${ }^{13}$ showed that the integral in the classical cross section, $d \sigma_{c} / d \epsilon$, could be expressed in terms of error functions. Equation (14) obviously reduces to the classical cross section in the limit of classical statistics$\eta B \rightarrow 1$ and $B \rightarrow \infty$.

\section{ELASTIC SCATTERING}

For $\epsilon=0$, the expression (12) for the cross section reduces to an indeterminate form and L'Hospital's rule must be used. In this case, the cross section is given by a simpler expression

$$
\begin{aligned}
& \frac{d^{2} \sigma_{ \pm}^{(e 1)}}{d \Omega d \epsilon}=(\eta B)^{-1}\left[1 \mp B^{-1} \exp \left(-\frac{1}{2}\left(\beta E_{i}\right) \frac{m}{M}(1-\cos \theta)\right)\right]^{-1} \\
& \times \frac{d^{2} \sigma_{c}^{(\mathrm{el})}}{d \Omega d \epsilon}
\end{aligned}
$$

where

$$
\begin{aligned}
\frac{d^{2} \sigma_{c}^{(\mathrm{el})}}{d \Omega d \epsilon}=\frac{1}{2}\left(\frac{\beta M}{2 \pi m E_{i}(1-\cos \theta)}\right)^{1 / 2} & \\
& \times \exp -\left(\frac{1}{2}\left(\beta E_{i}\right) \frac{m}{M}(1-\cos \theta)\right)
\end{aligned}
$$

and

$$
E_{i}=\left(\hbar^{2} k_{i}^{2} / 2 m\right) \text {. }
$$

Again, the behavior of the quantum-statistical cross section in the classical limit is obviously correct.

It is possible to obtain the following rather curious approximate expression for the integral of the cross

${ }^{18}$ E. P. Wigner and J. E. Wilkins, Jr., U.S. Atomic Energy Commission Rept. No. AECD-2275 (1944). section over solid angle:

$\left.d \sigma_{ \pm}{ }^{(\mathrm{el})} / d \epsilon \simeq(M / m)\left(4 \beta E_{i}\right)^{-1}\left\langle E^{-1}\right\rangle ; \quad\left[(m / M) \beta E_{i}\right\rangle 1\right]$, where $\left\langle E^{-1}\right\rangle$ denotes the reciprocal of the kinetic energy per scattering particle, averaged over the appropriate (BE or FD) distribution function.

\section{HELIUM-4 AS AN IDEAL QUANTUM GAS}

A practical approximation to an ideal quantum gas would, of course, be one of the noble gases. A quick examination shows that only ${ }^{4} \mathrm{He}$ (boson) and ${ }^{3} \mathrm{He}$ (fermion) exist in the gaseous form at temperatures low enough to permit values of $\eta$ large enough such that Eqs. (12) and (13) might give significantly different results for the cross section. This possibility was investigated for ${ }^{4} \mathrm{He}$ for two values of $\eta, \eta_{1}=0.1$ and $\eta_{2}=1$. The corresponding values of $B$, obtained from Ref. 3, are (for bosons) $B_{1}=10.4$ and $B_{2}=1.43$. The temperature chosen was $6^{\circ} \mathrm{K}$ because it is just above the critical temperature for ${ }^{4} \mathrm{He}, 5.2^{\circ} \mathrm{K}$, and at this temperature the fluid ${ }^{4} \mathrm{He}$ can be pressurized to $>200$ atm before solidifying. Since $\eta \sim \rho T^{-3 / 2}$, this avenue provides the largest possible values of $\eta$ for ${ }^{4} \mathrm{He}$ still in the gaseous phase. The density corresponding to $\eta_{1}$ and $T=6^{\circ} \mathrm{K}$ is $\rho_{1}=2.24 \times 10^{21}$ atoms $/ \mathrm{cm}^{3}$ and for $\eta_{2}$, $\rho_{2}=22.4 \times 10^{21} \mathrm{~cm}^{-3}$. The latter value is slightly greater than the density of liquid ${ }^{4} \mathrm{He}$ at the normal boiling point. The solid density at $6^{\circ} \mathrm{K}$ however is $\sim 40 \times 10^{21}$ $\mathrm{cm}^{-3}$. Thus both choices of parameters correspond to an ${ }^{4} \mathrm{He}$ system in the gaseous phase, but there is considerable doubt whether the gas could be considered ideal. To check this, a virial expansion of the form

$$
P=(A / V)+\left(A B_{v} / V^{2}\right)+\left(A C_{v} / V^{3}\right)
$$

was examined. The coefficients for ${ }^{4} \mathrm{He}$ at $6^{\circ} \mathrm{K}$ are

$$
\begin{aligned}
& A=2.1964 \times 10^{-2} \mathrm{~atm} ; \\
& B_{v}=-2.19 \times 10^{-8} ; \\
& C_{v}=0.91 \times 10^{-6},
\end{aligned}
$$

where $P$ is in atmospheres and $V$ is expressed as the ratio of the volume of a certain mass of gas to the volume of the same mass of gas at $1 \mathrm{~atm}$ and $0^{\circ} \mathrm{C} .^{14}$ The first term on the right-hand side of Eq. (18) is the ideal-gas law. Thus the magnitude of the second term provides an estimate of the extent of departure of the gas from an ideal gas. For $\eta_{1}=0.1$, Eq. (18) yields a pressure of $1.5 \mathrm{~atm}$ with the second term being an $18 \%$ correction. For the second value of $\eta, \eta_{2}=1$, Eq. (18) gives a negative value for the pressure, indicating that the pressure is beyond the region for which the expansion coefficients hold. Hence, for $\eta_{1}=0.1$, $T=6^{\circ} \mathrm{K}$, ${ }^{4} \mathrm{He}$ approximates an ideal gas, but for $\eta_{2}=1$, $T=6^{\circ} \mathrm{K}$, it is obviously far from being an ideal gas even though it still is in the gaseous phase.

${ }^{14}$ Argon, Helium, and the Rare Gases G. A. Cook, Ed. (Interscience Publishers, Inc., New York, 1961), Vol. 1. 
The cross section was calculated from both Expressions (12) and (13) for both sets of parameters, each for several values of $\epsilon$ and $\theta$. The incident neutron energy was taken as $0.005 \mathrm{eV}$. The result was that for $\eta=0.1$, Eqs. (12) and (13) never differed by more than $\sim 5 \%$, and that was for $\cos \theta=0.9$. For right-angle scattering, the differences were on the order of $1 \%$. For $\eta=1$, however, differences ranged from $\sim 40 \%$ for $\cos \theta=0.9$ to $\sim 15 \%$ and less for $\cos \theta=0$.

\section{CRITERION FOR IGNORING STATISTICS}

The condition obtained by insisting that $d^{2} \sigma_{ \pm} / d \Omega d \epsilon$ [as given by Eq. (12)] be equal to $d^{2} \sigma_{c} / d \Omega d \epsilon$ [as given by Eq. (13)] is (with the restriction, for bosons, that $\eta<2.612)$.

$$
\begin{aligned}
\eta= & \mp\left[e^{\alpha} /\left(e^{-\beta \epsilon}-1\right)\right] \\
& \times \ln \left\{1 \mp B^{-1} \exp [-(\alpha+\beta \epsilon)] /\left(1 \mp B^{-1} e^{-\alpha}\right)\right\} .
\end{aligned}
$$

For elastic scattering, the criterion is obtained from Eq. (16),

$$
\eta=\left\{B \mp \exp \left[-\frac{1}{2} \beta E_{i}(m / M)(1-\cos \theta)\right]\right\}^{-1} .
$$

The fractional amount by which the right-hand side of Eq. (19) differs from the value of $\eta$ for the ideal quantum gas scattering system in question is thus a direct measure of the correction to the neutron cross section due to symmetrization of the wavefunctions, for the scattering event characterized by the particular choice of momentum and energy transfer.

In deriving Eq. (19), the scatterer was assumed to be an ideal monatomic gas. However, this criterion for ignoring the statistics of the scatterer should give a rapid approximate estimate of the effect of statistics for weakly interacting systems as well.

\section{DISCUSSION}

In view of the nonintegrability of the boson and fermion distribution functions, it is interesting that the neutron cross section for scattering from an ideal quantum gas can be expressed in terms of well-known functions. It is true that, so far as the noble gases are concerned at least, one must conclude that all ideal gases obey Boltzmann statistics. However, it is quite apparent that this is far from true for all interacting gases.

The criterion of Eq. (19) is the same for polarized neutrons. This is because the effects of polarizing the neutron beam on the cross section are confined to the multiplicative constant which appears as a factor in both $d^{2} \sigma_{ \pm} / d \Omega d \epsilon$ and, naturally, after going to the classical limit, in $d^{2} \sigma_{c} / d \Omega d \epsilon_{\text {. }}$ Thus the effect cancels when the ratio is taken. What might accentuate the difference between $d^{2} \sigma_{ \pm} / d \Omega d \epsilon$ and $d^{2} \sigma_{c} / d \Omega d \epsilon$, if the ideal quantum gas in question had a nuclear magnetic moment, is to subject the scattering system to a magnetic field. Helium-3, with its spin of $\frac{1}{2} \hbar$ and its critical temperature lower even than that of ${ }^{4} \mathrm{He}$, would be a likely candidate for such a calculation, were it not for the matter of its huge absorption cross section for thermal neutrons.

\section{APPENDIX: DERIVATION OF EQS. (4) AND}

The object of this Appendix is to enable the reader who wishes to fill in the omitted steps between Eq. (2) and the equations (4) and (7) to do so with reasonable dispatch. However, because of the involved nature of the calculation, much detail still must be omitted. We outline the method and provide some essential formulas along with a few clarifying comments.

Let us consider separately the contributions to the right-hand side of Eq. (2) due to those terms with $j \neq l$ (the "distinct" terms) as opposed to those terms for which $j=l$ (the "self" terms).

$$
j \neq l:
$$

For $j \neq l$, the operator whose thermal average we seek is a two-particle operator which, when expressed in its second-quantized form, ${ }^{7}$ causes Eq. (2) to be written

$$
\begin{aligned}
&\left.\frac{d^{2} \sigma}{d \Omega d \epsilon}\right|_{\text {distinct }}=(2 \pi \hbar)^{-1} \frac{k_{f}}{k_{i}} \int_{-\infty}^{\infty} d t \exp \left(-i \frac{t}{\hbar} \epsilon\right)\left\langle\sum_{\mathbf{K}_{\alpha} \mathbf{K}_{\beta}, \mathbf{K}_{\gamma} \mathbf{K}_{\delta}} \sum_{m_{\alpha} m_{\beta}, m_{\gamma} m_{\delta}} A^{\dagger}\left(\mathbf{K}_{\alpha} m_{\alpha}\right) A^{\dagger}\left(\mathbf{K}_{\beta} m_{\beta}\right) A\left(\mathbf{K}_{\gamma} m_{\gamma}\right) A\left(\mathbf{K}_{\delta} m_{\beta}\right)\right. \\
& \times L^{-\alpha}\left\langle\exp \left(i \mathbf{K}_{\alpha} \cdot \mathbf{r}_{l}\right) \exp \left(i \mathbf{K}_{\beta} \cdot \mathbf{r}_{j}\right) y\left(m_{\alpha}\right) y\left(m_{\beta}\right)\right| a_{j}^{\dagger} a_{l} \exp \left(-i \mathbf{Q} \cdot \mathbf{r}_{j}\right) \\
&\left.\left.\quad \times \exp \left[i \mathbf{Q} \cdot\left(\frac{t}{m} \mathbf{p}_{l}+\mathbf{r}_{l}\right)\right]\left|y\left(m_{\gamma}\right) y\left(m_{\delta}\right) \exp \left(i \mathbf{K}_{\gamma} \cdot \mathbf{r}_{j}\right) \exp \left(i \mathbf{K}_{\delta} \cdot \mathbf{r}_{l}\right)\right\rangle\right\rangle\right\rangle_{T}
\end{aligned}
$$

$A^{\dagger}(\mathrm{K} m)$ is the creation operator which gives rise to a particle with momentum $\hbar \mathrm{K}$ and spin projection $m$; $A(\mathbf{K} m)$ is the corresponding annihilation operator. $L^{3}$ is the volume of the scattering sample. The $y(m)$ are actually $\left(2 S_{0}+1\right) \times 1$ column matrices specifying the spin state of the nucleus in question. The sums on $\mathbf{K}_{\alpha}$, etc., and $m_{\alpha}$, etc., run over all cells in momentum and spin space. We have also used the fact that for an ideal gas

$$
\mathbf{r}_{l}(t)=(t / m) \mathbf{p}_{l}+\mathbf{r}_{l}(0) .
$$


One uses the operator identity

$$
e^{A} e^{B}=e^{A+B} \exp \left(\frac{1}{2}[A, B]\right),
$$

which is valid if $[A, B]$ commutes with both $A$ and $B$, then works out the part of the matrix element between spatial states with the result that the matrix element within the thermal average in (A1) becomes

$$
\begin{aligned}
& \exp [(i t / \hbar)\left.\left(E_{\mathbf{K}_{\alpha}}-E_{\mathbf{K}_{\alpha}-\mathbf{Q}}-\epsilon\right)\right] \\
& \times \delta\left(\mathbf{K}_{\delta}-\mathbf{K}_{\alpha}+\mathbf{Q}\right) \delta\left(\mathbf{K}_{\gamma}-\mathbf{K}_{\beta}-\mathbf{Q}\right) \\
& \quad \times\left\langle m_{\alpha} m_{\beta}\left|a_{j}^{\dagger} a_{l}\right| m_{\gamma} m_{\delta}\right\rangle .
\end{aligned}
$$

The factor of $L^{-6} \exp [-i(\epsilon / \hbar) t]$ in (A1) has been absorbed in (A3).

Next we calculate

$$
\left\langle i\left|A_{\alpha}^{\dagger} A_{\beta}^{\dagger} A_{\gamma} A_{\delta}\right| i\right\rangle,
$$

where we have compressed the notation by writing $A^{\dagger}\left(\mathbf{K}_{\alpha} m_{\alpha}\right)$ as $A_{\alpha}{ }^{\dagger}$, etc. Because of the properties of the creation and destruction operators and the orthogonality of the number density states $|i\rangle$, the nonvanishing contributions occur only for two cases:

$$
\begin{array}{ll}
\text { (1) } \alpha=\gamma, & \beta=\delta, \\
\text { (2) } \alpha=\delta, & \beta=\gamma .
\end{array}
$$

We note further the following commutation rules which the creation and destruction operators must obey:

$$
\left[A_{\alpha}, A_{\beta}^{\dagger}\right]_{\mp}=\delta_{\alpha \beta}, \quad\left[A_{\alpha}, A_{\beta}\right]_{\mp}=\left[A_{\alpha}^{\dagger}, A_{\beta}^{\dagger}\right]_{\mp}=0 .
$$

(The commutation relations obtain for $\mathrm{BE}$ statistics whereas the anticommutation laws hold for FD statistics.) One can thus write

$$
\begin{aligned}
\left\langle i\left|A_{\alpha}{ }^{\dagger} A_{\beta}{ }^{\dagger} A_{\gamma} A_{\delta}\right| i\right\rangle & =\left\{ \pm\left\langle i\left|A_{\alpha}^{\dagger} A_{\alpha} A_{\beta}^{\dagger} A_{\beta}\right| i\right\rangle \mp \delta_{\alpha \beta}\left\langle i\left|A_{\alpha}^{\dagger} A_{\alpha}\right| i\right\rangle\right\}\left\{\delta_{\alpha \gamma} \delta_{\beta \delta} \pm \delta_{\alpha \delta} \delta_{\beta \gamma}\right\} \\
& = \pm\left\{n_{\alpha}^{(i)} n_{\beta}^{(i)}-n_{\alpha}{ }^{(i)} \delta_{\alpha \beta}\right\}\left\{\delta_{\alpha \gamma} \delta_{\beta \delta} \pm \delta_{\alpha \delta} \delta_{\beta \gamma}\right\} .
\end{aligned}
$$

The $n_{\alpha}{ }^{(i)}$ is the occupation number of the $\alpha$ th cell in the momentum-spin space of the scattering particles when the scattering system is in the state $|i\rangle$, i.e., $n_{\alpha}{ }^{(i)}$ is an eigenvalue of the number operator $A_{\alpha}{ }^{\dagger} A_{\alpha}$ which is diagonal in the number density representation. As usual, our sign convention is that upper signs refer to BE statistics, lower signs to FD statistics. Equation (A5) is actually in error due to the omission of a term which would correct for double counting the contribution from $\alpha=\beta=\gamma=\delta$; however, since one would find that such a contribution ultimately turns out to be small at least by a factor of $N$, the number of scattering nuclei, we leave it out of (A5). For precisely the same reason, the contribution included in (A5) proportional to $\delta_{\alpha \beta} \delta_{\alpha \gamma} \delta_{\beta \delta}$ is ignored also (as one might guess since this will reduce the sum $\sum_{\alpha, \beta, \gamma, \delta}$ to $\sum_{\alpha}$ compared with other terms which go as $\sum_{\alpha, \beta}$ ). Thus the expression for the distinct contribution to the cross section can be written

$$
\begin{aligned}
& d^{2} \sigma /\left.d \Omega d \epsilon\right|_{\text {distinot }}= \pm\left(k_{f} / k_{i}\right) \sum_{\alpha, \beta, \gamma, \delta} \delta\left(E_{\mathrm{K}_{\alpha}}-E_{\mathrm{K}_{\alpha}-\mathrm{Q}}-\epsilon\right) \delta\left(\mathbf{K}_{\delta}-\mathbf{K}_{\alpha}+\mathbf{Q}\right) \delta\left(\mathbf{K}_{\gamma}-\mathbf{K}_{\beta}-\mathbf{Q}\right) Z^{-1} \\
& \quad \times \sum_{i} \exp \left(-\beta E_{i}\right) n_{\alpha}^{(i)} n_{\beta}^{(i)}\left\{\delta_{\alpha \gamma} \delta_{\beta \delta} \pm \delta_{\alpha \delta} \delta_{\beta \gamma}\right\}\left\langle m_{\alpha}\left|b_{1}\left\langle\chi_{f} \mid \chi_{i}\right\rangle+b_{2} \boldsymbol{\delta} \cdot \mathbf{S}\right| m_{\delta}\right\rangle\left\langle m_{\beta}\left|b_{1}^{*}\left\langle\chi_{f} \mid \chi_{i}\right\rangle^{*}+b_{2}{ }^{*} \boldsymbol{d}^{*} \cdot \mathbf{S}\right| m_{\gamma}\right\rangle,
\end{aligned}
$$

where

$$
E_{i}=\sum_{\alpha} n_{\alpha}^{(i)}\left(\hbar^{2} K_{\alpha}^{2} / 2 M\right)=\sum_{\alpha} n_{\alpha}^{(i)} E_{\mathrm{K}_{\alpha}}
$$

and

$$
Z=\sum_{j} \exp \left(-\beta E_{j}\right)
$$

Because of the identical nature of the scatterers, the indices $j$ and $l$ no longer indicate a meaningful distinction and have been dropped. The summation notation has been compressed with $\sum_{\alpha}$ actually meaning $\sum_{\mathrm{K}_{\alpha}} \sum_{m_{\alpha}}$, etc. Finally, the integral representation of the Dirac energy conservation delta function

$$
\delta(E)=(2 \pi \hbar)^{-1} \int_{-\infty}^{\infty} \exp \left(i \frac{t}{\hbar} E\right) d t
$$

has been recognized as such. Equation (A6) is correct even for the $B E$ gas in the condensed phase, in which case the terms we have neglected either are still negligible or ultimately cancel.

In order to calculate the matrix elements between spin states, one writes the $x$ and $y$ components of $\mathrm{S}$ in terms of the familiar raising and lowering operators $S_{+}$and $S_{-}$:

$$
\mathbf{S}=\frac{1}{2}\left(S_{+}+S_{-}\right) \hat{z}+\left[\left(S_{+}-S_{-}\right) / 2 i\right] \hat{\jmath}+S_{Z} \hat{k},
$$

where $\hat{\imath}, \hat{\jmath}$, and $\hat{k}$ are the unit vectors in the $x, y$, and $z$ directions, respectively, and

$$
S_{ \pm}\left|S_{0}, m\right\rangle=\left[S_{0}\left(S_{0}+1\right)-m(m \pm 1)\right]^{1 / 2}\left|S_{0}, m \pm 1\right\rangle .
$$

(Here the \pm signs have no connection with statistics!) 
One finds that

$$
\begin{aligned}
\sum_{m_{\alpha} m_{\beta}, m_{\gamma} m_{\delta}}\left\langle m_{\alpha}\left|b_{1}\left\langle\chi_{f} \mid \chi_{i}\right\rangle+b_{2} \mathbf{d} \cdot \mathbf{S}\right| m_{\delta}\right\rangle\left\langle m_{\beta}\left|b_{1}^{*}\left\langle\chi_{f} \mid \chi_{i}\right\rangle^{*}+b_{2}{ }^{*} \boldsymbol{d}^{*} \cdot \mathbf{S}\right| m_{\gamma}\right\rangle \\
\times\left\{\begin{array}{l}
\delta_{m_{\alpha} m_{\gamma} \delta_{m_{\beta} m_{\delta}}} \\
\delta_{m_{m m_{\delta}} \delta_{m_{\beta} m_{\gamma}}}
\end{array}=\left\{\begin{array}{l}
\left(2 S_{0}+1\right)\left[\left|b_{1}\right|^{2}\left|\left\langle\chi_{f} \mid \chi_{i}\right\rangle\right|^{2}+\left|b_{2}\right|^{2}|\sigma| \frac{1}{3} S_{0}\left(S_{0}+1\right)\right] \\
\left(2 S_{0}+1\right)^{2}\left|b_{1}\right|^{2}\left|\left\langle\chi_{f} \mid \chi_{i}\right\rangle\right|^{2} .
\end{array}\right.\right.
\end{aligned}
$$

Finally one has to do the averages over the density matrix:

$$
Z^{-1} \sum_{i} \exp \left(-\beta E_{i}\right)() \equiv\langle()\rangle
$$

These averages can be shown to be, for an ideal gas (with zero external magnetic field) of either fermions or bosons, except for bosons in the condensed phase (until this point, our results have applied to all bosons and fermions),

$$
\begin{aligned}
\left\langle n_{\mathrm{K} m}\right\rangle & =\left\langle n_{\mathrm{K}}\right\rangle /\left(2 S_{0}+1\right) \\
\left\langle n_{\mathrm{K}^{\prime}} n_{\mathrm{K}^{\prime} m^{\prime}}\right\rangle & =\left\langle n_{\mathrm{K}}\right\rangle\left\langle n_{\mathrm{K}^{\prime}}\right\rangle /\left(2 S_{0}+1\right)^{2}
\end{aligned}
$$

except for

$$
\mathrm{K}=\mathrm{K}^{\prime}, \quad m=m^{\prime}
$$

in which case

$$
\left\langle n_{\mathrm{Km}}^{2}\right\rangle=\left\langle n_{\mathrm{K}}\right\rangle /\left(2 S_{0}+1\right) .
$$

In Eq. (A8), $\left\langle n_{\mathrm{K}}\right\rangle$ is simply $f_{\mathrm{K}}^{\mp}$ as given by Eq. (5). Perhaps the simplest way to derive (A8) is to perform the averages over the density matrix in the grand canonical ensemble by the familiar device of taking the appropriate derivatives of the grand partition function. The treatment differs from those conventionally given in statistical mechanics books which do not treat the spin-dependent case.

For an ideal gas of bosons characterized by a value of $\eta \geq 2.612$, the gas can be treated as a mixture of two different noninteracting phases. For the phase in the ground $(\mathbf{K}=0)$ state, the averages of (A8) are not valid and must be replaced by

$$
\begin{aligned}
\left\langle n_{0 m}\right\rangle & =N_{0} /\left(2 S_{0}+1\right), \\
\left\langle n_{0 m^{2}}{ }^{2}\right. & =2 N_{0}^{2} /\left(2 S_{0}+1\right)\left(2 S_{0}+2\right), \\
\left\langle n_{0 m} n_{0 m^{\prime}}\right\rangle & =N_{0}^{2} /\left(2 S_{0}+1\right)\left(2 S_{0}+2\right) ; \quad m \neq m^{\prime} .
\end{aligned}
$$

The problem represented by the bosons in the ground state is no more than the occupancy problem in probability theory of $N_{0}$ identical particles and $2 S_{0}+1$ boxes. The results assume zero external magnetic field, and that $N_{0}^{2} \gg N_{0}$.

The other phase consists of those bosons with nonzero energy and for this phase, the averages of (A8) are valid.

Using (A8) one is able to complete the calculation of the distinct contribution to the cross section as it appears in Eq. (4). For the condensed-phase BE gas, one must work a bit harder because of the need to calculate "mixed" averages like $\left\langle n_{\mathrm{O} m} n_{\mathrm{K} m^{\prime}}\right\rangle$, which however can be expressed in terms of (A8) and (A9). Also, the argument which one must make (and is easy to justify for $\left.N_{0} \ll N\right)$ allowing one to ignore $\sum_{\mathrm{K} \neq 0}\left\langle n_{\mathrm{K}}\right\rangle^{2}$ compared with $N_{0}^{2}$ requires a bit more justifying.

Much of the difficulty of the calculation can be eliminated if one chooses to ignore forward scattering. The decision not to do so arises from a desire to obtain the constant term in $\Gamma(\mathbf{r}, t)$. In working toward $\mathrm{Eq}$. (7), one must be concerned with the fact that $\left\langle n_{0 m} n_{0 m^{\prime}}\right\rangle$ does not reduce to $\left\langle n_{0 m}{ }^{2}\right\rangle$ for $m=m^{\prime}$ (although this is also true for $\mathbf{K} \neq 0$, a correction term for this failing will turn out to be down by a factor of $N$ and hence utterly negligible) and, therefore, one has to tack on the correct contribution including a factor of $\delta(Q)$ since such a term contributes only to the forward scattering. One should not subtract out the incorrect contribution in a like manner because it does not survive the four-dimensional Fourier transformation to $\Gamma(\mathbf{r}, t)$ anyway since $d^{3} \mathrm{Q}=\mathrm{Q}^{2} d \mathrm{Q} d \Omega$ vanishes for $\mathrm{Q}=0$ and hence washes out any $Q=0$ contribution from a term not containing an explicit factor of $\delta(\mathrm{Q})$.

$$
j=l \text { : }
$$

The "self"-contribution to the cross section is more tractable, partly because it develops that the condensedphase BE gas does not need to be treated as a separate case. One simply observes that the second-quantized form of the operator [which must be thermal averaged in Eq. (2) with $j=l]$

$$
\sum_{j=1}^{N} a_{j}^{\dagger} a_{j} \exp \left(i \frac{t}{m} \mathbf{Q} \cdot \mathbf{p}_{j}\right)
$$

is

$$
\sum_{\alpha, \beta} A_{\alpha}^{\dagger} A_{\beta} L^{-3}\left\langle\exp \left(i \mathbf{K}_{\alpha} \cdot \mathbf{r}_{j}\right) y\left(m_{\alpha}\right)\left|a_{j}^{\dagger} a_{j} \exp \left[i(t / m) Q \cdot \mathbf{p}_{j}\right]\right| \exp \left(i \mathbf{K}_{\beta} \cdot \mathbf{r}_{j}\right) y\left(m_{\beta}\right)\right\rangle
$$

and proceeds from there as he did in the previous case of $j \neq l$. When the self-contribution to the cross section is added to the appropriate distinct contribution, one then obtains both Eqs. (4) and (7). 